\title{
European hake (Merluccius merluccius) assessment based on size frequencies and basic biological parameters in the SW Mediterranean
}

\author{
Pedro Martínez-Baños ${ }^{a}$, John Gabriel Ramírez ${ }^{b}$, Montserrat Demestre ${ }^{b}$, Francesc Maynou ${ }^{b}$ \\ ${ }^{a}$ C \&C - MEDIO AMBIENTE, C. Antonio Oliver n 17, Ed. Spica, 3A, 30204, Cartagena, Spain \\ b Institut de Ciències del Mar, CSIC, Psg. Marítim de la Barceloneta 37-49, 08003, Barcelona, Spain
}

\section{Introduction}

Many target species, as well as most stocks in multispecies fisheries, do not meet the requirements for assessment using data-rich methods. These species, by default, are usually considered data limited. However, defining, for example, how much the fishing mortality is above or below a reference point requires appropriate knowledge of life-history traits (Kokkalis et al., 2015; Magnusson and Hilborn, 2007; Ramirez et al., 2017). The spawning potential ratio can be estimated from length-based techniques using data-limited methods (Hordyk et al., 2015c). The performance of this method depends on the accurate estimates of life history ratios, and it is especially sensitive to the estimates of $L^{\infty}$ (Hordyk et al., 2015c). I addition, Prince et al. (2015) found that the most uncertain assessments were derived from small size samples.

The stock assessments of hake in the northwest and southwest Mediterranean Sea by working groups of the STECF or the GFCM in recent years have relied on the fastgrowth hypothesis that was principally justified from a tagrecapture experiment in the Gulf of Lions (Mellon-Duval et al., 2010) which determined two different growth curve Consequently, we examined the sensitivity of the results of the LB-SPR method to a range of $L \infty$ and $k$ values from 100.7 to $110 \mathrm{~cm}$ and from 0.112 to $0.236 \mathrm{y}-1$, respectively ("slow" and "fast" growth hypotheses). The hake stock in the Murcia region (southwest Mediterranean Sea) provide high-quality data for estimating year-to-year length-based SPR values. Therefore, the objective of this work is to assess the exploitation rate of hake in the SW Mediterranean based on a size frequency analysis of individuals sampled from commercial catches over 13 years using the LB-SPR method under different assumptions and uncertainties of life-history parameters.

\section{Methods}

Hake were measured onboard commercial trawlers working on hake fishing grounds (50-350m depth approximately) in the Murcia region from April to December 1998 to 2010 from 85 fishing trips. The sampled data were weighted by catch in number by depth stratum and month to obtain a representative annual size distribution. We measured 31,969 hake with sizes ranging between 5 and $74 \mathrm{~cm}$ TL. The modal value was $16.5 \mathrm{~cm} \mathrm{TL}$, and $95 \%$ of individuals were between 7 and $37.5 \mathrm{~cm}$ TL. Length frequency data after 2010 were not considered because of regulatory mesh changes in Mediterranean trawls (adoption of $40-\mathrm{mm}$ square meshes) that could imply changes in catchability.

Hake is not fished with fishing gear other than bottom trawls in the Murcia region. There are two contrasting hypotheses regarding hake growth in the Mediterranean, with $\mathrm{k}$ values of approximately $0.1 \mathrm{yr}-1$ for the slow-growth hypothesis and $k$ values of approximately $0.2 \mathrm{yr}-1$ for the fast-growth hypothesis (Table 1). Additional uncertainties in the SPR outputs were quantified by placing distributions bounded by upper and lower values of $L \infty$, the CV of $L \infty, M / k, L 50$ and L95 to account for the uncertainties of these parameters. Assuming that the length frequency data are the only available information, we explored the F/M and SPR estimates by grouping the annual length frequencies in four periods (1998-2000, 2001-2003, 2004-2006 and 20072010) (Fig. 1). Thus, we provided a smoothed trend of the LBSPR estimates to reduce the potential effect of the annual non-equilibrium state.
Main results

- The similarity of the annual estimates of the SPR under the four life history parameters depended strongly on how well the model fitted the size data, while the SPR estimates were not sensitive to the growth hypotheses.

- The concept of the fast- or slow-growth hypothesis virtually disappears when $\mathrm{M} / \mathrm{k}$ instead of $\mathrm{M}$ and $\mathrm{k}$ separately is used. Thus, two growth hypotheses produced fairly similar $\mathrm{M} / \mathrm{k}$ values (Table 1).

- The absence of large individuals in the size frequency would not result in large underestimates of the SPR if selectivity was not dome-shaped. However, we cannot rule out the possibility of dome-shaped selectivity, i.e., the apparent absence of large individuals from bottom traw catches due to variation in catchability for biological reasons.

- Even though our sample sizes were larger than 1000 by year, the SPR estimates from the four life-history paramete sets varied among years (from $1 \%$ to $6 \%$ approx.) (Fig. 2). In the framework of fisheries management using a harvest control rule even small changes to the current SPR could mean additional time to return to sustainable levels because more than one recovery trajectory may occur.

The years when the four life-history sets produced virtually the same SPR values were derived from large samples ( 2000 individuals) and corresponded to most of the best fits to the annual length distributions (Fig 2).

- Regardless which of the four life-history parameter sets was used, the SPR was never higher than $8 \%$ during the study period. Comparable SPR values were obtained accounting for the uncertainties of the estimates of $L \infty$, the $C V$ of $L \infty, M / k$ and maturity-at-size (Fig. 3). Moderate changes in recruitment, which probably led to transitory population size structures, did not prevent the reliable SPR estimates for hake.

Table 1. von Bertalanffy growth parameters and natural mortality of hake in the Mediterranean Sea used in this study. M was estimated using PRODBIOM from the average M at ages $0-5$ (Abella et al, 1997$)$.

\begin{tabular}{|c|c|c|c|c|c|c|c|}
\hline Source & Region & Sex & $L_{\mathrm{s}}(\mathrm{cm})$ & $M\left(\mathrm{yr}^{-1}\right)$ & $K\left(\mathrm{yr}^{-1}\right)$ & Growth hypothesis & $M / k$ \\
\hline Mellon-Duval et al. (2010), model 3 & Gulf of Lions & Male, female, undetermined & 110 & 0.565 & 0.178 & Fast & 3.17 \\
\hline Mellon-Duval et al. (2010), model 1 & Gulf of Lions & Female & 100.7 & 0.62 & 0.236 & Fast & 2.63 \\
\hline Aldebert and Recasens (1996) & Catalan Sea & Female & 100.7 & 0.29 & 0.124 & Slow & 2.34 \\
\hline Martínez-Baños et al. (Data in Brief) & Murcia & Combined & 105.87 & 0.34 & 0.112 & Slow & 3.04 \\
\hline
\end{tabular}

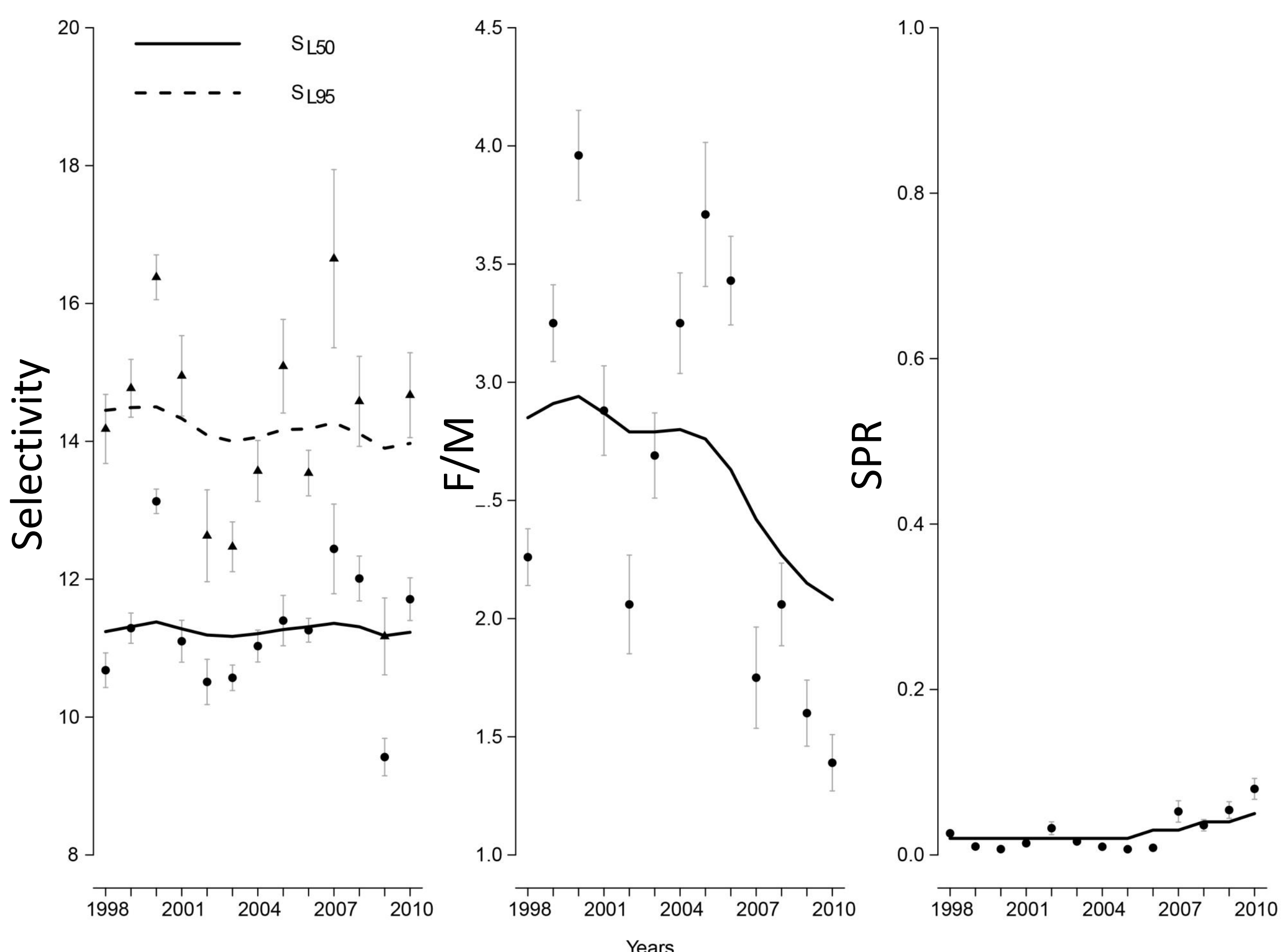

Figure 1. LB-SPR outputs for the life-history parameters estimated for the Murcia selectivity, F/M and SPR. Confidence intervals (95\%) are shown in vertical bars.

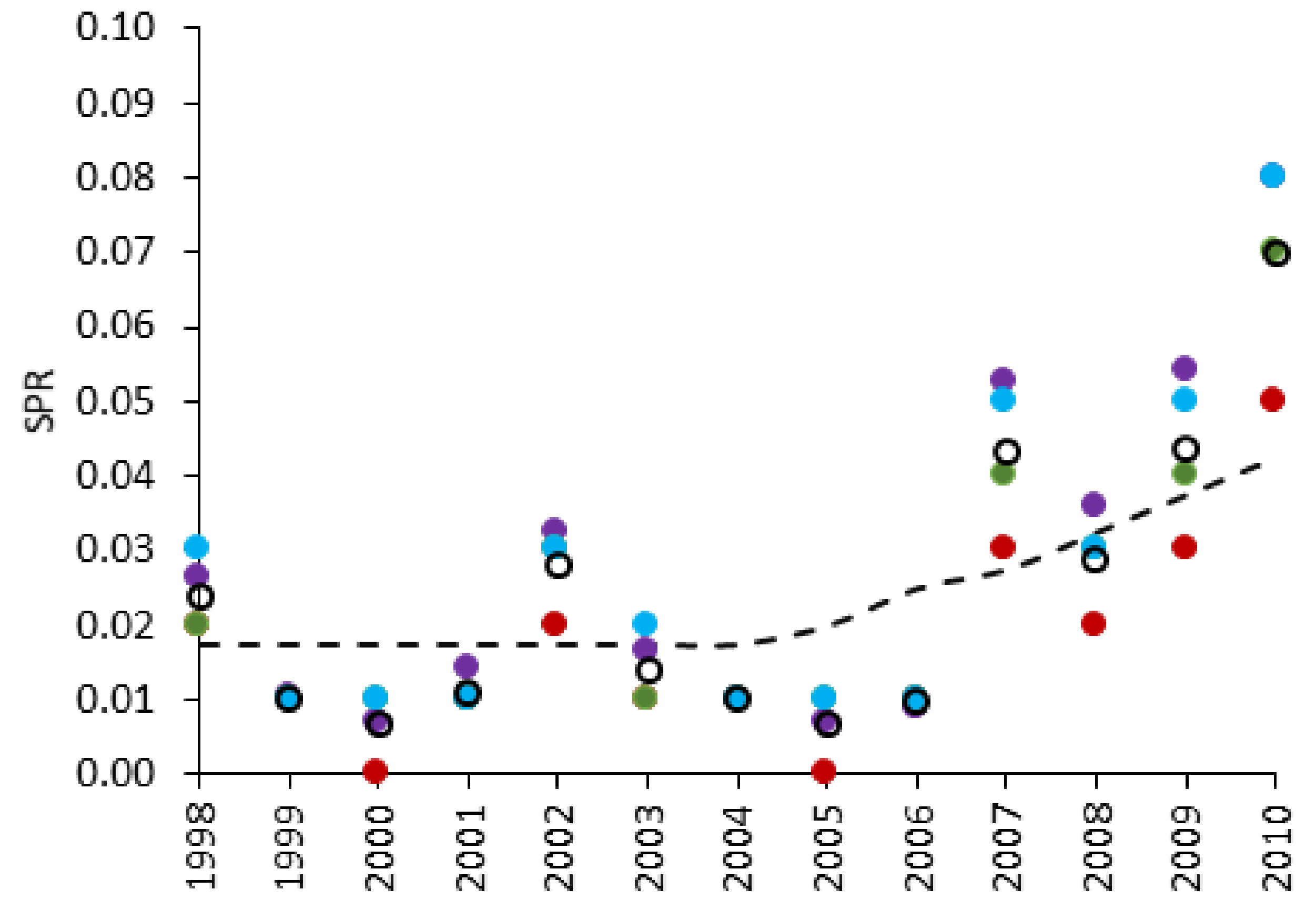

Figure 2. SPR estimated using growth parameter estimated during this study (purple circles), by Aldebert and Recasens (1996) (red circles), from model 1 of Mellon-Duval et al. (2010) (green circles), and from model 3 of Mellon-Duval et al. (2010) (blue circles). Mean are shown.

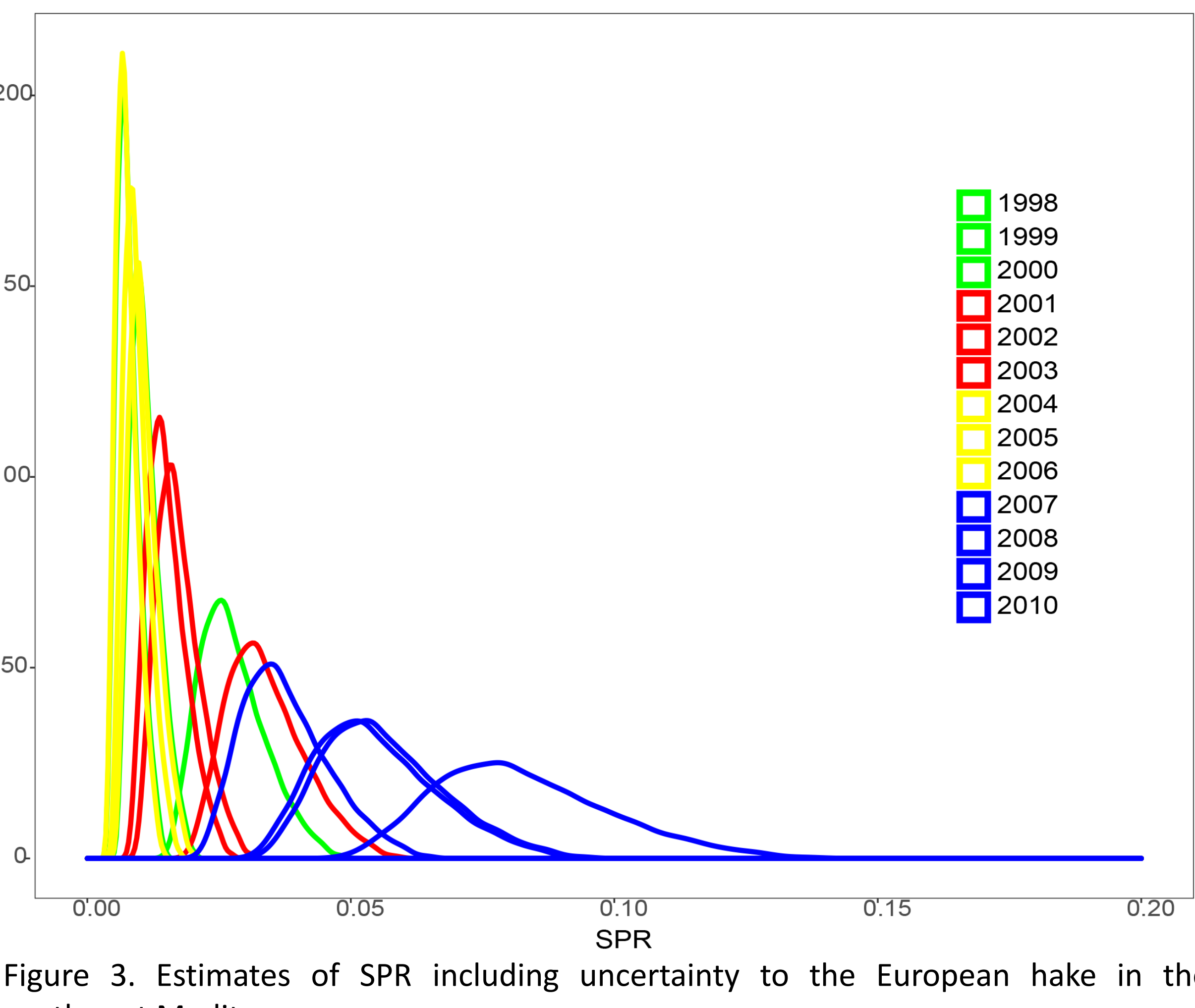

southwest Mediterranean. 